\title{
Research on Dynamic Effects of Employability of Vocational College Students in Taiwan
}

\author{
Pin-Chang Chen \\ Department of Information Management, Yu Da University of Science and Technology, \\ Miaoli, Taiwan
}

\begin{abstract}
This study used the dynamic scenario simulation of system dynamics to perform simulation on the effects of education policies, trend of employment demand, and employability qualities of vocational college graduates on the development of employment demand in Taiwan's technology industry. According to the research results, dynamic situational simulation of system dynamics can be used to simulate the effect of changes in education policy system with time on the development and trend of employment demand in technology industry. The simulation results of policy scenario showed that the talent shortage in technology industry should be overcome by improving education policy. The problem of talent shortage cannot be effectively alleviated until the matching rate between education policy and employment demand reaches 90\%. The simulation results of this study can be provided as reference for education policy planners to improve the employability of vocational college students. It is intended to provide valuable suggestions to reduce the unemployment rate of vocational college graduates and to substantially reduce the gap between industries and academia, in order to further enhance Taiwan's competitiveness in the global economic system.
\end{abstract}

\section{KEYWORDS}

System Dynamics, Employability, Vocational Education

\section{INTRODUCTION}

In Taiwan, vocational education has played a very important role in the cultivation of technical personnel for a long time. It has cultivated many outstanding technical personnel with strong competitiveness to meet the need for technical improvement and industrial upgrading. It has also promoted Taiwan's international industrial competitiveness and created Taiwan's economic miracle [1].

With the transformation of global economic environment, the industrial structure of Taiwan has been gradually changed from the past labour-intensive traditional industries to the current technology and capital-intensive technology industry [2]. However, after the vocational education in Taiwan was highly expanded, the level of vocational college students becomes inconsistent. Vocational college students cannot exert their professional competence, and thus to meet industrial specifications, which leads to the imbalanced situation between vocational college graduates' abilities and abilities required by workplace [3]. Therefore, there are the so-called phenomena of "enterprises fail to find talents," "underachievement of gifted students," "one's education does not fit him for a certain job" and "underemployment of graduates." According to the results of many surveys, the cause of manpower shortage of current enterprises is not the failure to find people, but the failure to find "suitable" talents. Therefore, current education policy

DOI : 10.5121/ijcsit.2014.6113 
should meet the needs of industries. In addition to "teaching students in accordance with their aptitude," education policies should also meet the trends and aim to cultivate the talents that the current industries need [4].

Therefore, this study is intended to use the advantages of dynamic simulation of system dynamics to combine the education policies in Taiwan and trend of employment demand of technology industry with the employability quality of vocational college graduates. It is hoped that valuable suggestions can be made for the future vocational education policies in Taiwan.

\section{LITERATURE REVIEW}

The concept of system dynamics originates from cybernetics. System dynamics is intended to be used as a management tool. In static studies, system will not change with time. It is impossible to analyse the complicated changes of system structure with time. As a result, this study is intended to use the management concepts of cause-and-effect feedback relationship of system dynamics to investigate various effects developed in the overall system to respond to the changes in external factors/conditions with time. Because system dynamics can be used to effectively resolve the deficiency that it is difficult to quantify variables in general calculation and analysis model and dynamic environment. Therefore, the system status simulated from system dynamics will be able to tally with real situation of real environment [5].

In early days, system dynamics were firstly applied to production and distribution problems. The famous example was published in the book "Limits to Growth" by Forrester which simulated five variables including global population, food, resource consumption, industrialization and contamination in the future world. The results showed that, global resources can support economic growth and development to a certain limits. If countermeasures are not proposed early, disastrous consequences will appear in the future [6]. Doyle et al. applied the model developed using system dynamics to understanding the profits of promotional costs and balancing the real profits of sales promotion. System dynamics can be applied to a wide scope of fields, including organizational policies, industrial economy, management function, global issues, etc. System dynamics theory has become a matured and useful methodology [7]. Dangerfield used system dynamics model to simulate the spread of AIDS in the Europe and the establishment of medial policies [8]. Lyneis and Ford proposed four kinds dynamic structures of simulation project. The project features model was developed using the features of system dynamics to simulate the real system model. The main components of real project were used to develop the model, strengthen the ability to simulate the dynamics of real project and connect the experiences of project managers to increase the accuracy of decision-making [9]. Han et al. used system dynamics model to develop the model of sustainable urban development in China, and used the model simulation to understand the effects of urban development and expected policies on long-term urban development [10]. Ceresia used system dynamics simulation to predict the optimal configuration for improving organizational performance in the issues of objective management and human resources [11].

According to the investigations of the aforementioned studies, system dynamics possesses the advantage of dynamic simulation, and is applied to various fields to reach the conclusion that is close to real situation. Therefore, this study chose to use system dynamics to perform dynamic simulation on education policies, trend of employment demand of technology industry and employability qualities of vocational college graduates. Moreover, this study provided the simulation results for education policy planners as reference and suggestions to enable vocational 
education to cultivate the manpower meeting the needs of industries, in order to reduce the gap between industries and academia.

\section{MODEL DEVELOPMENT}

This study used Vensim software of system dynamics to perform dynamic simulation on gaps among education policies in Taiwan, trend of employment demand in technology industry and employability qualities of vocational college graduates. This study determined related variables as Causal Feedback Loop Diagram and then includes relevant cause-and-effect variables in the Stock-Flow Diagram to develop the system dynamics model [12].

The Causal Feedback Loop Diagram of this study is show in Figure1.

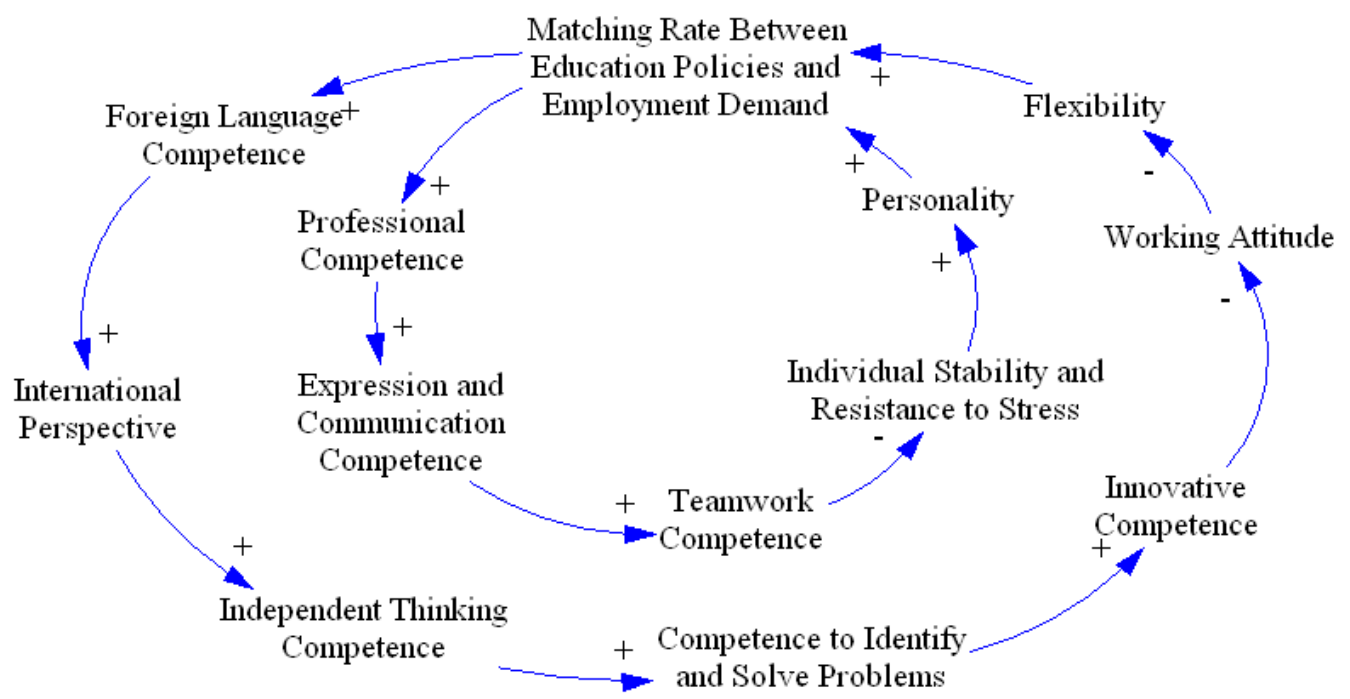

Figure 1. Causal Feedback Loop Diagram

When the matching rate between education policies and employment demand is high, professional competence possessed by graduates can better meet the employment demand of enterprises. However, graduates with sufficient professional competence can get along with people more smoothly in workplace and enhance their teamwork competence after receiving complete expression and communication training. However, the pros and cons of teamwork competence cannot fully represent the level of individual stability and resistance to stress. Therefore, personality traits can be changed through education policies to further increase individual stability and resistance to stress.

According to the causal feedback loop diagram, this study developed the Stock-Flow Diagram as shown in Figure 2. 


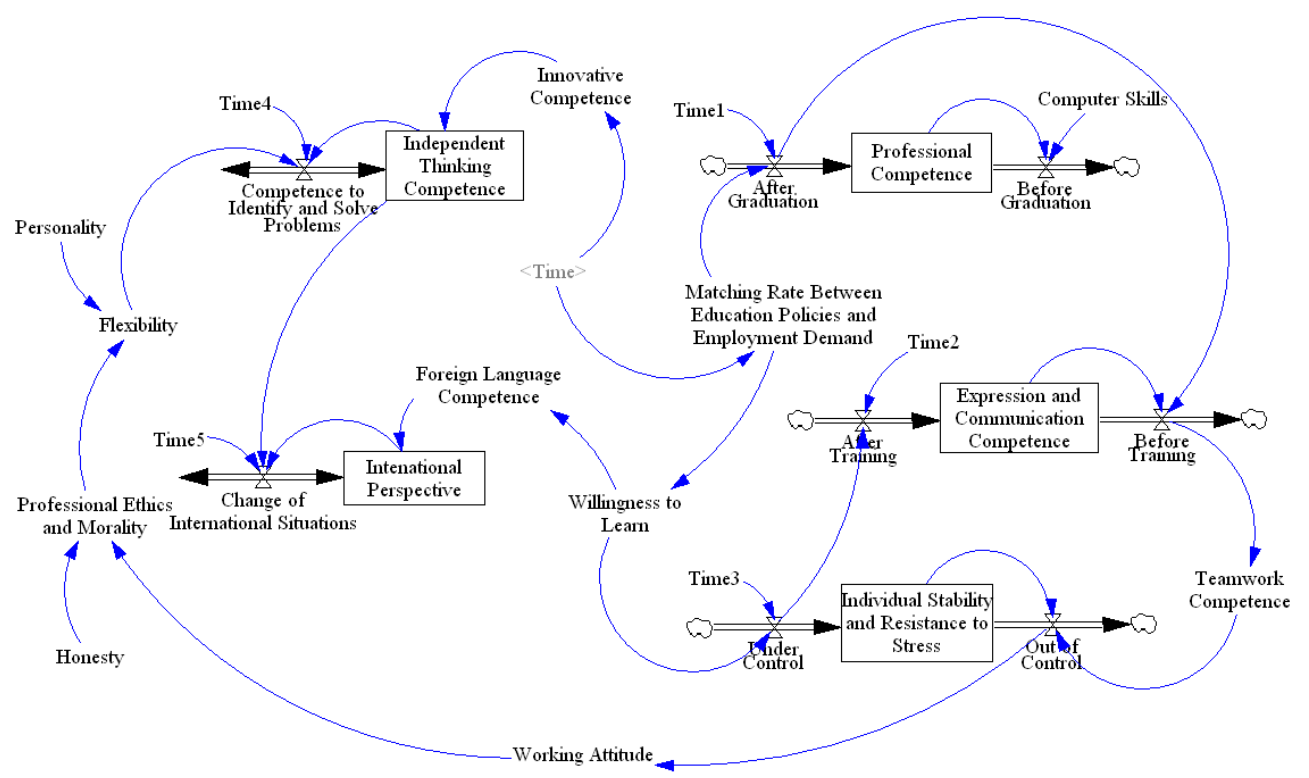

Figure 2. Stock-flow Diagram

Current education policies in Taiwan have attached a lot of importance to students' foreign language competence in order to enhance students' international perspective. Moreover, such education policies also aim to improve vocational education graduates' independent thinking competence. After graduates' independent thinking competence is improved, their competence to identify and solve problems can also be improved to further increase individual innovative competence. Moreover, enterprises also attach importance to employees' working attitude. However, the employees possessing the aforementioned competences many not necessarily possess a good working attitude. Besides, the flexibility of employees with a good working attitude may not necessarily be good. Therefore, employees' flexibility still has to be gradually cultivated through the overall national education policies.

\section{RESUlTS AND DISCUSSION}

This study simulated the model of the variable "matching rate between education policies and employment demand." This study observed the priority considerations of employment and their changes in technology industry by probing into the matching rate between education policies and employment demand and the employability required to be possessed by vocational education graduates.

This study chose to use three policy scenarios of high (90\%), medium (60\%) and low (30\%) matching rate between education policies and employment demand to perform the simulation on the future 3-year gap between trend of employment demand in technology industry and employability qualities of vocational education graduates. This study first adjusted two variables including matching rate between education policies and employment demand and post-graduation professional competence, and then compared the conditions such as independent thinking, innovative competence, expression and communication competence, foreign language competence and international perspective to prove that education policies are inseparable from the cultivation of future industrial talents of a country. The simulation results of policy scenarios in this study are described as follows: 


\subsection{Policy Scenario 1}

This study set the conditions as follows: high matching rate (90\%) between education policies and employment demand, post-graduation professional competence (50\%), willingness to learn $(80 \%)$ and working attitude $(80 \%)$ to perform simulation, as shown in Figure 3.

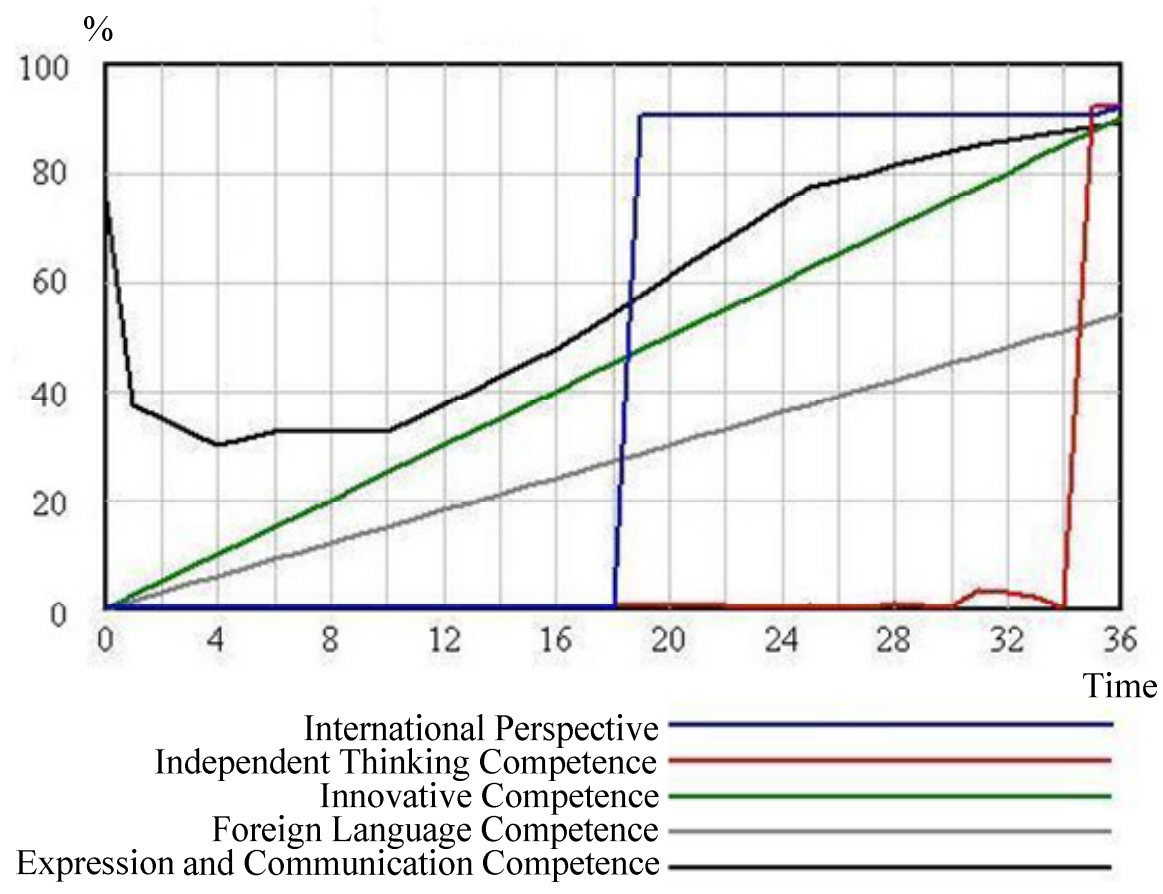

Figure 3. Trend of Effect of High Matching Rate between Education Policies and Employment Demand on Vocational College Students' Employability

As shown in Figure 3, although the conditions of students in various aspects were not ideal in the beginning of implementation of education policies, their foreign language competence gradually improved and their international perspective also increased to $90 \%$ in Month 18 after receiving the training for a period of time. With the improvement in international perspective, independent thinking competence was further inspired, and rapidly increased to $91 \%$ in Month 34 . The expression and communication competence and innovative competence also increased to $90 \%$. In addition, the five conditions of employment demands mentioned above all increased by at least 52\% after Month 36. Enterprise employers not only found the talents they needed, but also reduced the cost of personnel training as well as enhanced corporate industrial competitiveness.

\subsection{Policy Scenario 2}

This study set the conditions as follows: medium matching rate $(60 \%)$ between education policies and employment demand, post-graduation professional competence (50\%), willingness to learn $(80 \%)$ and working attitude (80\%) to perform simulation, as shown in Figure 4. 


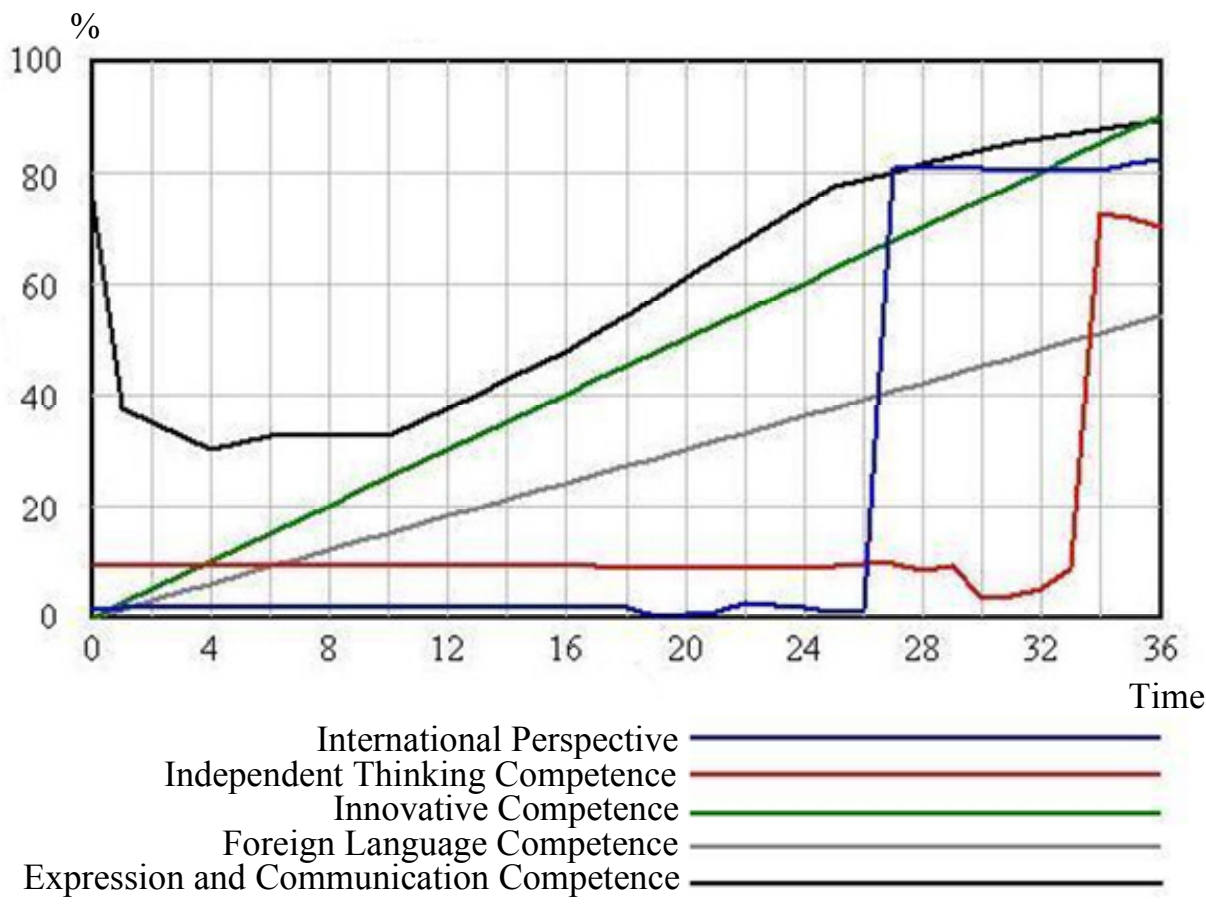

Figure 4. Trend of Effect of Medium Matching Rate between Education Policies and Employment Demand on Vocational College Students' Employability

As shown in Figure 4, if there was no higher matching rate between education policies and employment demand, the improvement in students' international perspective would be prolonged for 8 months, compared to that of policy scenario 1. In addition, it increased at least $80 \%$ in 3 years. Independent thinking competence reached the highest point of $73 \%$ in Month 34, and then started to decline. As a result, this policy scenario did not lead to the steady improvement in conditions of employment demands of graduates, which increased the cost of personnel training as well as further obstructed corporate industrial competitiveness.

\subsection{Policy Scenario 3}

This study set the conditions as follows: low matching rate (30\%) between education policies and employment demand, post-graduation professional competence (50\%), willingness to learn (80\%) and working attitude (80\%) to perform simulation, as shown in Figure 5. 


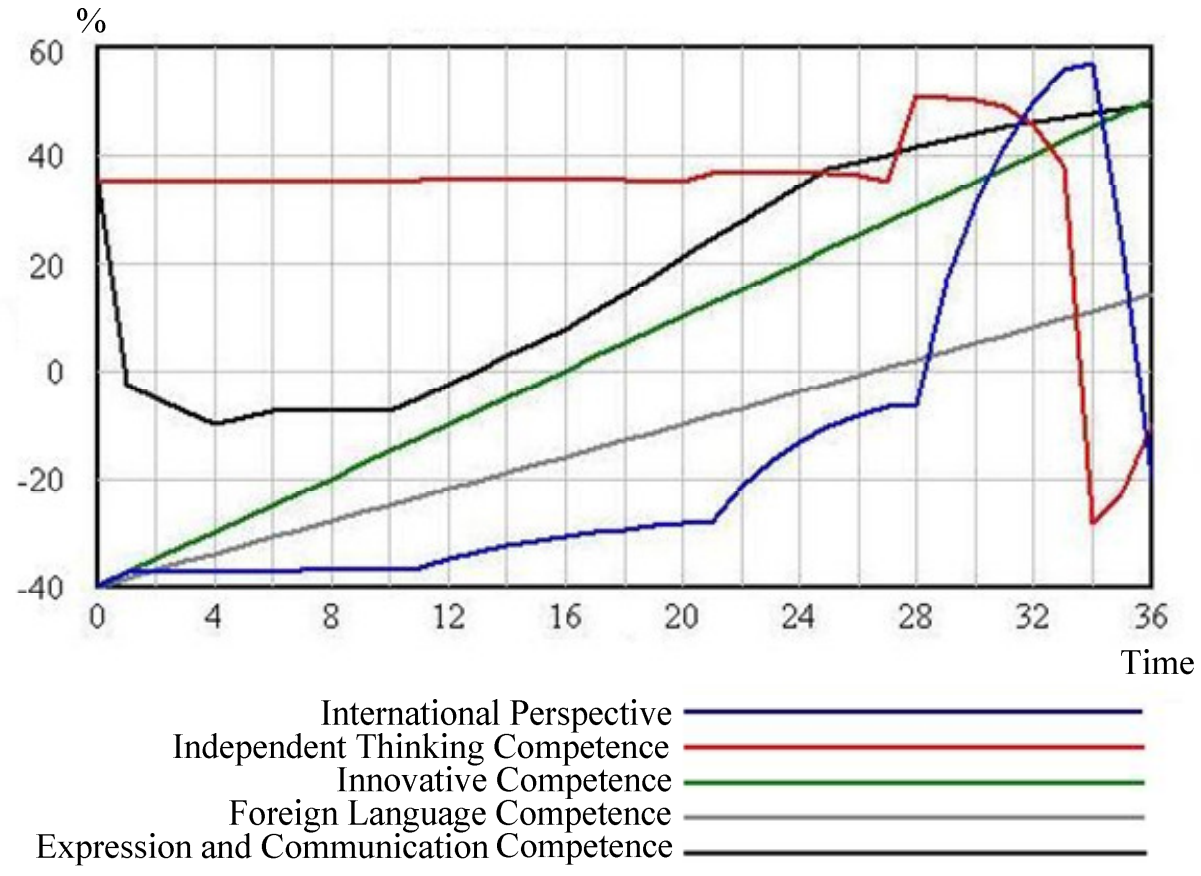

Figure 5. Trend of Effect of Low Matching Rate between Education Policies and Employment Demand on Vocational College Students' Employability

As shown in Figure 5, in the beginning of the promotion of education policies, students all aggressively participated in educational activities. Therefore, their foreign language and international perspective both improved constantly. However, after a period of time, due to the lack of constant promotion and implementation of subsequent policies, the independent thinking competence declined significantly in Month 30, and international perspective also rapidly declined in Month 34. Therefore, if the gap between education policies and employment demand was huge, the original level of students' competences as required by enterprises could not be maintained and would even decline after a period of time. Consequently, such education policies were not feasible.

In summary, the simulation results of policy scenario showed that the talent shortage in technology industry should be overcome by improving education policy. The problem of talent shortage cannot be effectively alleviated until the matching rate between education policy and employment demand reaches $90 \%$. Education policies should be developed according to the overall employment demand to help improve the future employability of vocational education students, as well as to reduce the unemployment rate of the current college graduates. In addition, developing education policies according to employment demand can also cultivate the talents needed by enterprises to reduce the gap between industries and academia and to further improve Taiwan's global economic competitiveness.

\section{CONCLUSION}

The advantage of the past technology industry in Taiwan was sufficient higher education talents. However, with the transformation of global economic environment, there have been no national boundaries in talent transfer. The technical talents in Taiwan will face strict challenges from 
various places around the world. Under such a situation, the enterprises lacking in talents with innovative competence, international perspective and independent thinking will face serious threats.

To improve overall national economic competitiveness, it is necessary to strengthen the cultivation of talents with innovative competence, international perspective and independent thinking competent through education policies. Moreover, workplace experiencing provided by the cooperation between schools and enterprises or vocational training institutions enables students to learn by practicing and try to integrate theories with practices. It can better trigger students' innovative competence and competence to identify and solve problems, which is the most important current issue in Taiwan.

In addition, the policy simulation model developed in this study can be further modified into a more complete model based on the transformation of global economic environment and interactions among industries to make the overall Stock-Flow Diagram more complete. The stimulation results can be provided more accurately as important reference for future researchers to promote policies.

\section{REFERENCES}

[1] Huang, H. I. \& Lee, C. F., (2012) "Strategic management for competitive advantage: a case study of higher technical and vocational education in Taiwan", Journal of Higher Education Policy and Management, Vol. 34, No. 6, pp. 611-628.

[2] Mo, H. E. \& Chen, Y. F., (2011) "An empirical study on the renaming departments for higher technological and vocational education in Taiwan", The Journal of American Academy of Business, Vol. 16, No. 2, pp. 270-278.

[3] Chang, T. Y. \& Hsu, J. M., (2010) "Development framework for tourism and hospitality in higher vocational education in Taiwan”, Journal of Hospitality, Leisure, Sport \& Tourism Education, Vol. 9, No. 1, pp. 101-109.

[4] Chou, C. M., (2010) “Analysis of college students' satisfaction with educational functions and teaching patterns of technological and vocational education in Taiwan", World Transactions on Engineering and Technology Education, Vol. 8, No. 3, pp. 262-267.

[5] Sterman, J. D., (2000) Business dynamics: Systems thinking and modelling for a complex world, Boston, USA: Irwin McGraw-Hill.

[6] Forrester, J. W., (1971) World dynamics, Cambridge, USA: The M.I.T. Press.

[7] Doyle, P., Saunders, J. \& Wong, V., (1986) "A comparative investigation of Japanese marketing strategies in the UK," Journal of International Business Studies, Vol. 17, No. 1, pp. 27-46.

[8] Dangerfield, B. C., (1999) "System dynamics applications to European health care issue," Journal of the Operational Research Society, Vol. 50, No. 4, pp. 345-353.

[9] Lyneis, J. M. \& Ford, D.N., (2007) "System dynamics applied to project management: a survey, assessment, and directions for future research," System Dynamics Review, Vol. 23, No. 2-3, pp. 157189.

[10] Han, J., Hayashi, Y., Cao, X. \& Imura, H., (2009) "Application of an integrated system dynamics and cellular automata model for urban growth assessment: A case study of Shanghai, China”, Landscape and Urban Planning, Vol. 91, No. 3, pp. 133-141.

[11] Ceresia, F., (2009) "A model of goal dynamics in organizations: a case study", The 27th International Conference of the System Dynamics Society, Albuquerque, New Mexico, USA.

[12] Eberlein, R. L. \& Peterson, D. W., (1992) "Understanding models with Vensim”, European Journal of Operational Research, Vol. 59, No. 1, pp. 216-219. 